American Journal of Applied Sciences 7 (6): 846-851, 2010

ISSN 1546-9239

(C) 2010 Science Publications

\title{
Sessile Drop Evaporation and Leidenfrost Phenomenon
}

\author{
A.K. Mozumder, M.R. Ullah, A. Hossain and M.A. Islam \\ Department of Mechanical Engineering, \\ Bangladesh University of Engineering and Technology, Dhaka 1000, Bangladesh
}

\begin{abstract}
Problem statement: Quenching and cooling are important process in manufacturing industry for controlling the mechanical properties of materials, where evaporation is a vital mode of heat transfer. Approach: This study experimentally investigated the evaporation of sessile drop for four different heated surfaces of Aluminum, Brass, Copper and Mild steel with a combination of four different liquids as Methanol, Ethanol, Water and $\mathrm{NaCl}$ solution. The time of evaporation for the droplet on the hot metallic surface was measured and compared with a proposed correlation as well. With the time temperature plot of these experimental data, the Leidenfrost phenomena had been elucidated. In the pool boiling curve for liquid, just after the transition boiling region and before the film boiling region, the heat transfer approaches its minimum value. The corresponding temperature of this minimum value was termed as the Leidenfrost temperature and the phenomenon is known as Leidenfrost phenomena. According to the experimental data, the Leidenfrost temperature was within a range of $150-200^{\circ} \mathrm{C}$ for all the experimental conditions. Results: This revealed that Leidenfrost temperature was independent of thermo-physical properties of solid and liquid. Sessile drop evaporation time was the maximum for water, then decreases gradually for Nacl solution, methanol and was the minimum for ethanol for a particular solid material. On the other hand, this time was the highest for copper and the lowest for mild steel for a specific liquid. Conclusion: The experimental data for the evaporation time fairly agree with the proposed correlation within a certain range. The collected time and temperature data may be used as a good data bank for the researchers.
\end{abstract}

Key words: Evaporation, sessile drop, conduction

\section{INTRODUCTION}

When a liquid drop falls upon a hot solid surface, an insulating vapor layer is immediately formed between the droplet and hot surface which results in decrease of heat transfer than the case of direct contact.

Many studies of the Leidenfrost phenomenon have already been appeared in the literature. The droplet evaporation process after impinging on a solid wall near Leidenfrost point was theoretically analyzed by Heng and Zhou (2007). A correlation for predicting evaporation lifetime was obtained based on the theoretical analysis and experimental results. Gottfried et al. (1966) analyzed evaporation time data for small droplet of five ordinary liquids and proposed an analytical model which was in fair agreement with the data. The model postulates that heat is transferred to the droplet by conduction from the plate below the droplet through the supporting vapor film and by radiation from the plate; mass is removed by diffusion from the outer surface and by evaporation from the lower surface.
Michiyoshi and Makino (1978) investigated the heat-transfer characteristics for evaporation of droplet of pure water placed on smooth surfaces of copper, brass, carbon steel and stainless steel at temperature ranging from $80-450^{\circ} \mathrm{C}$. They correlated the heattransfer with temperature. A numerical investigation for the evaporation process of $\mathrm{n}$-heptane and water droplets impinging onto a hot substrate was conducted by Nikolopoulos et al. (2007). The evaporation rates of droplets of n-heptane and water were also investigated by Elyssa and Black (2004) which showed that the trends in the wetted diameter, height and contact angle for water were fundamentally different from heptane.

Literature reveals that according to the definition of Leidenfrost temperature, it varies approximately from $600-800^{\circ} \mathrm{C}$ for pool boiling. In the present study, for sessile drop evaporation, the value of this temperature has been tried to found out. Among all of the experimental conditions, the Leidenfrost temperature varies from $150-200^{\circ} \mathrm{C}$ which is much smaller than the value of pool boiling. It is not an easy task to explain

Corresponding Author: A.K. Mozumder, Department of Mechanical Engineering,

Bangladesh University of Engineering and Technology, Dhaka 1000, Bangladesh 
this difference unless the mechanism of Leidenfrost phenomena is completely understood. Among several causes, vapor pressure might be one of them. In the case of pool boiling, a liquid column exists over the vapor layer which might increase the vapor pressure (and ultimately the boiling curve shifts to the right) and increase the temperature for minimum heat flux (Leidenfrost temperature). On the other hand, for a sessile drop evaporation, the weight of the drop let is very much negligible compared to a liquid column which is not capable to produce any extra pressure on the vapor layer, this relatively lower pressure consequences the Leidenfrost temperature to become smaller.

To obtain more insight into the phenomenon an investigation has been carried out concerning sessile drop evaporation. This study experimentally investigates the evaporation of sessile drop for four different heated surfaces of Aluminum, brass, copper and mild steel with a combination of four different liquids as methanol, ethanol, water and $\mathrm{NaCl}$ solution.

\section{MATERIALS AND METHODS}

Experimental procedure: The sessile drop apparatus was used to study the evaporation characteristics of droplet on a heated surface. In particular, the liquidsolid interface temperature corresponding to the Leidenfrost temperature was determined from droplet evaporation curve for different materials with different liquids.

The experimental setup consists of four metal blocks, one stand, two heaters, one variac, one thermocouple and one dropper (as shown in Fig. 1) The working fluids were water, $\mathrm{NaCl}$ solution, methanol and ethanol. Two 500 watt cartridge heaters were used to heat the metal block and they were placed beneath the test surface by drilling the block. K type ChromelAlumel thermocouple was used to determine the center temperature of the testing surface. The thermocouple was installed $3 \mathrm{~mm}$ below the test surface. Regulated electrical energy was supplied to the heater by using a variac, connected to the 220 volt laboratory power. A couple of syringes were used to drop the liquid droplets on the test surface. The syringe was held perpendicular to the horizontal test surface and droplets were released from about two inches from the surface. Though the test surface was little concave, it is assumed that heat is transferred to the droplet from a flat surface.

From the schematic of the experimental apparatus it is seen that heating surface was heated from the bottom by using two cartridge heaters. When the temperature reached a predetermined value (say $100^{\circ} \mathrm{C}$ ), a droplet was dropped to the center of the heating surface with a syringe; evaporation time was measured using a stopwatch. The droplet temperature was equal to the room temperature when it was dropped. The surface temperature was measured using a digital multimeter and a $1 \mathrm{~mm}$ diameter chromel-alumel (type $\mathrm{K}$ ) thermocouple located $3 \mathrm{~mm}$ beneath the center of the test surface. Few numbers of observed phenomena (dancing characteristics) of the droplet evaporation activities was captured using a video camera.

The droplet's initial diameter was calculated as a sphere from the measured average volume of 30 droplets. There might have a little error in measuring the diameter of the droplets.

A stopwatch was used to record the time of evaporation of droplets and its accuracy was $0.01 \mathrm{sec}$. To minimize the timer $( \pm 0.01 \mathrm{sec})$ and initial droplet size errors, three evaporation times were recorded for each temperature and then averaged together. This procedure was performed for $25^{\circ} \mathrm{C}$ surface temperature increment.

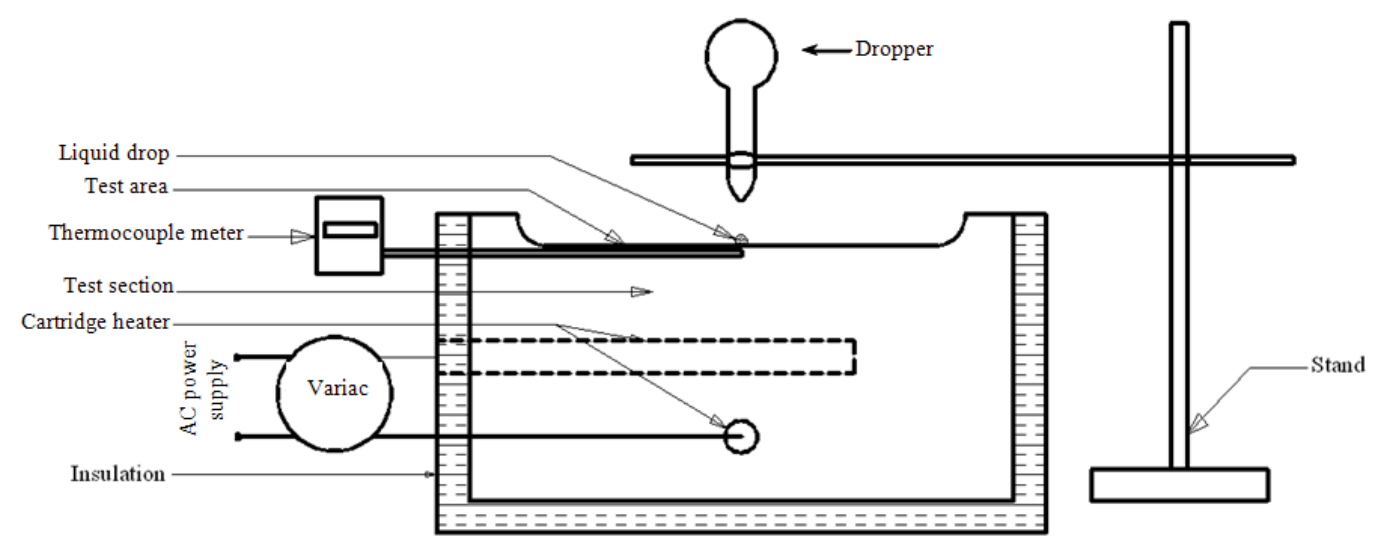

Fig. 1: Experimental setup 


\section{RESULTS AND DISCUSSION}

The experimental evaporation times are shown in Fig. 2-9. The temperature which gives maximum evaporation time is presumed to be the minimum heat flux at which stable film boiling can exist and is termed as Leidenfrost temperature. To the lower of the Leidenfrost Temperature, the boiling is in transition regime between nucleate and film.

From Fig. 2 it is shown that water stands out compared with the other liquids by virtue of having a much longer vaporization time. The Leidenfrost time for water and $\mathrm{NaCl}$ solution are nearly equal. On the other hand both methanol and ethanol shows small value of Leidenfrost time.

Figure 3 and 4 also show that the Leidenfrost time is almost same for methanol and ethanol. It is also evident from the graph that water shows the highest evaporation time among the four working fluids. For mild steel, these times are comparable Fig. 5.

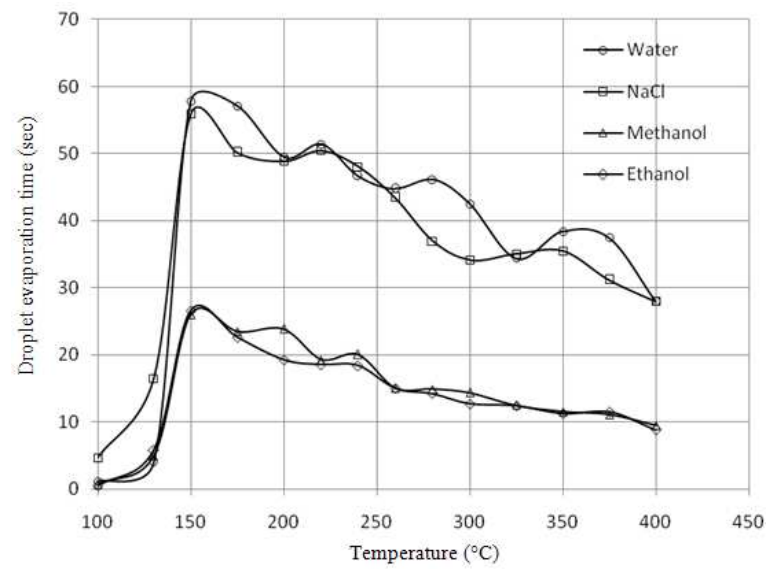

Fig. 2: Comparison of droplet evaporation time of water, $\mathrm{NaCl}$ solution, methanol, ethanol on aluminum

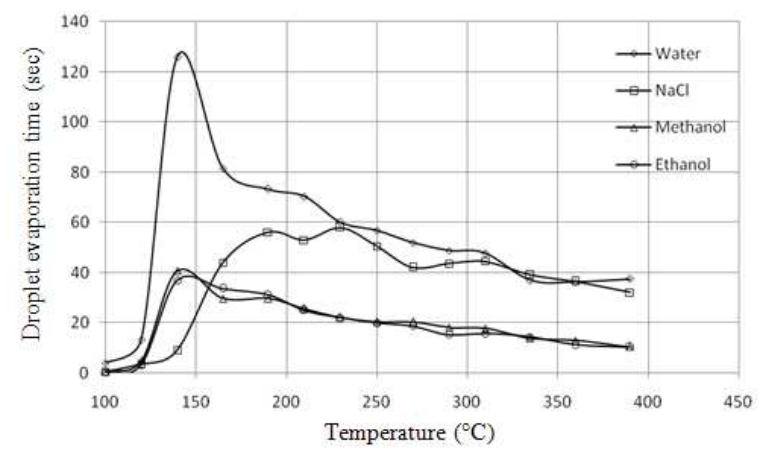

Fig. 3: Comparison of droplet evaporation time of water, $\mathrm{NaCl}$ solution, methanol, ethanol on brass
Leidenfrost temperature and time values were obtained for water, $\mathrm{NaCl}$ solution, methanol and ethanol on aluminum, copper, brass and mild steel surface. The Leidenfrost time is nearly identical for aluminum, brass and mild steel surfaces but is slightly higher for the copper surface (Bernardin and Mudawar, 1999). Figure 6 and 7 also shows the Leidenfrost times and temperatures for the four metal surfaces. The Fig. 6 reveals that materials with higher conductivity (aluminum, brass and copper) have longer Leidenfrost time than lower conductivity (mild steel) for $\mathrm{NaCl}$ solution. But for methanol (Fig. 7) all of them show comparable results where brass shows longer time then sequentially copper, aluminum and mild steel. Since copper has higher conductivity, it should take longer Leidenfrost time. But due to some uncertainty of the experiment, there might have a bit irregularity in the result. Further experimentation might provide much better results.

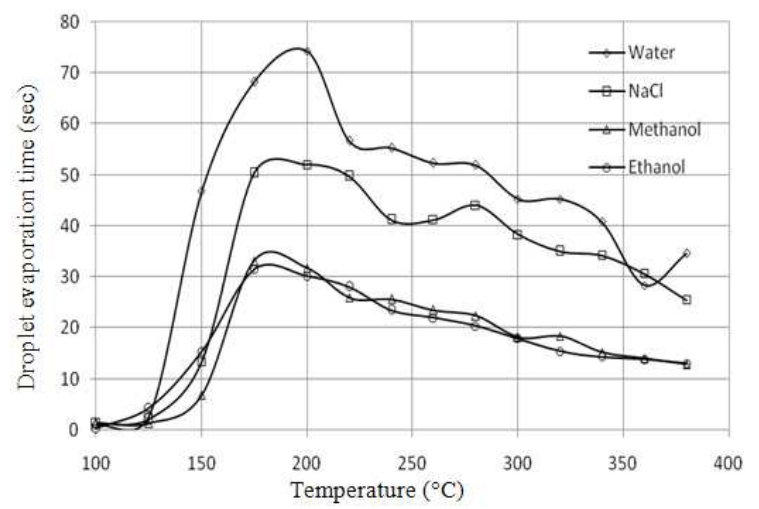

Fig. 4: Comparison of droplet evaporation time of water, $\mathrm{NaCl}$ solution, methanol, ethanol on copper

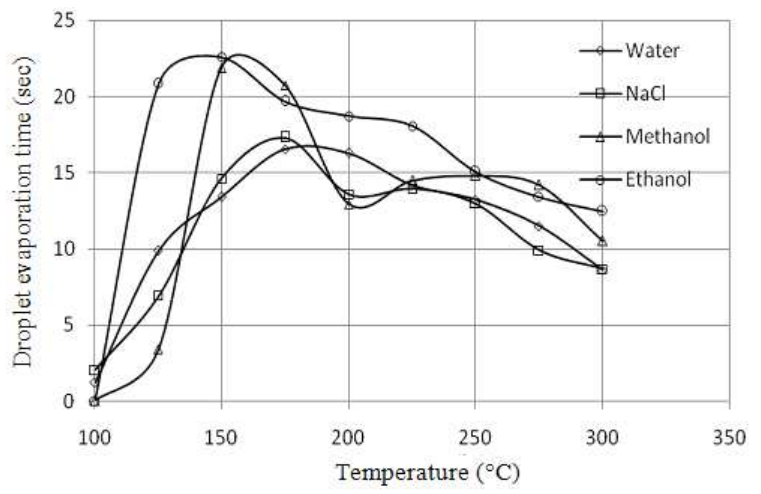

Fig. 5: Comparison of droplet evaporation time of water, $\mathrm{NaCl}$ solution, methanol, ethanol on mild steel 
Am. J. Applied Sci., 7 (6): 846-851, 2010

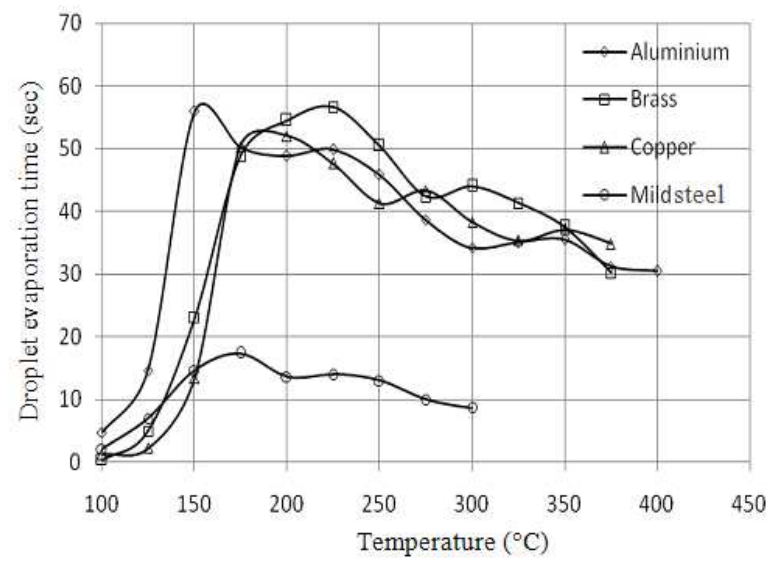

Fig. 6: Comparison of droplet evaporation time of $\mathrm{NaCl}$ on mild steel, aluminum, brass, copper

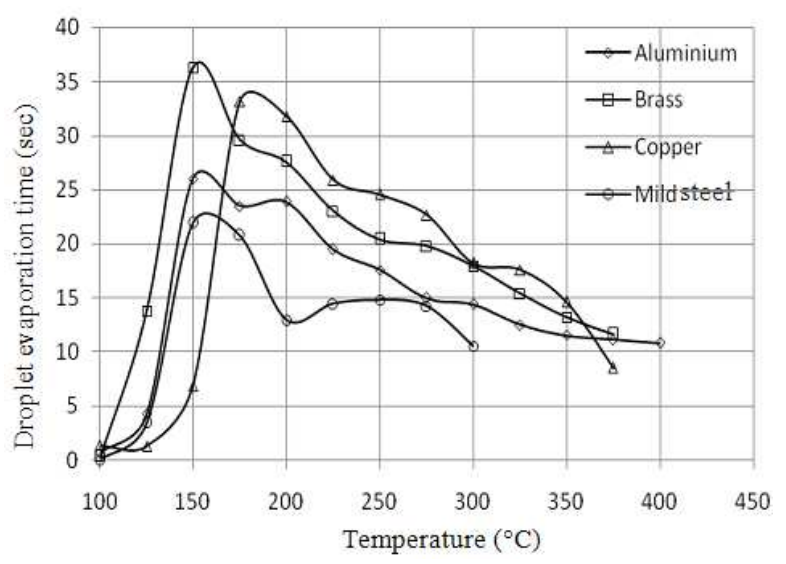

Fig. 7: Comparison of droplet evaporation time of methanol on mild steel, aluminum, brass, copper

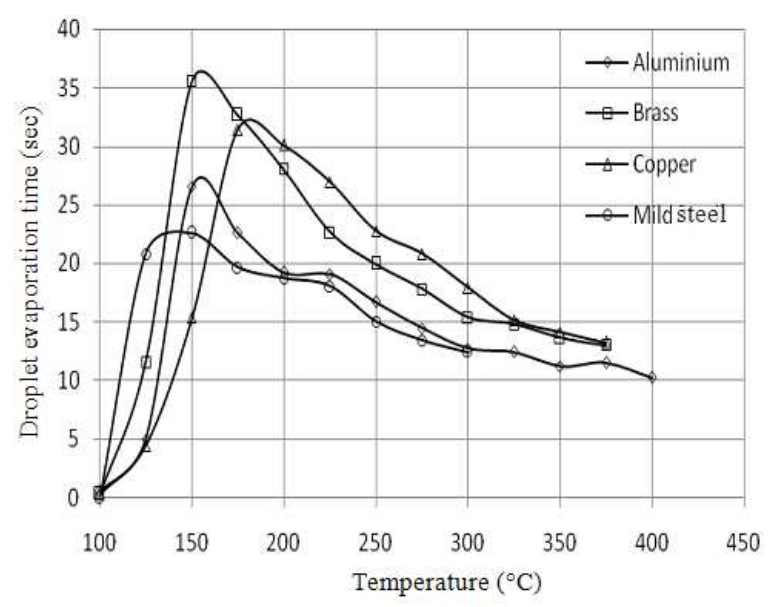

Fig. 8: Droplet evaporation time of ethanol on mild steel, aluminum, brass, copper

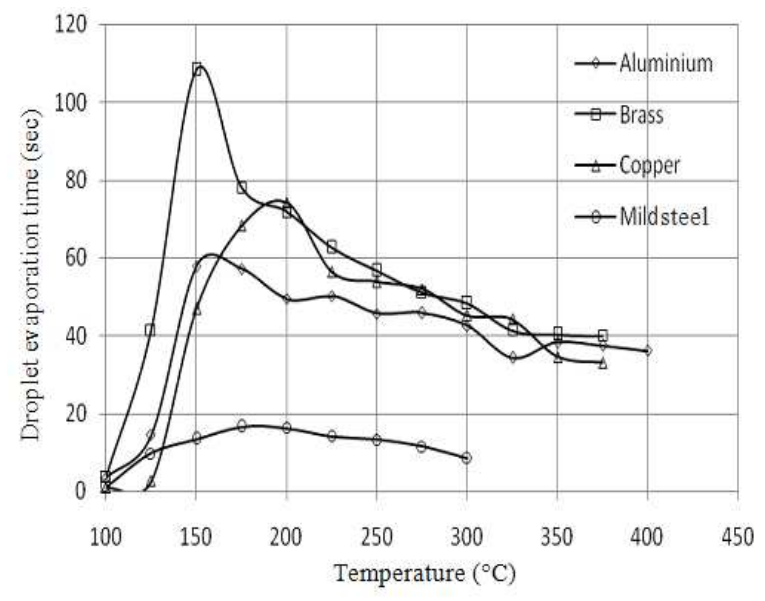

Fig. 9: Droplet evaporation time of water on mild steel, aluminum, brass, copper

The higher droplet evaporation time is speculated to be the result of higher conductivity than other metal. Higher conductivity means higher heat transfer through the metal. It means, when liquid touches the metal, large amount of vapor will produce due to higher heat transfer and the surface is completely covered by a vapor blanket and then heat transfer from the surface to the liquid occurs by conduction through vapor. Droplet supported by the vapor film slowly boils away.

Figure 8 and 9 shows the droplet evaporation time of ethanol and water on the four different metal surfaces. For both the working fluids, the evaporation time is the maximum for brass surface.

Development of correlation: It is postulated that several physical processes occur simultaneously over the upper and lower surfaces of the droplet. Heat is transferred to the droplet by conduction through the moving vapor film between the lower half of the droplet. Heat is also transferred by radiation from the hot surface. Mass is removed from the droplet by evaporation in to the vapor film on the lower surface and by diffusion controlled evaporation of the upper half surface.

The mass, heat and force balances for the liquid droplet must be satisfied. To write the necessary equation, the droplet is assumed spherical and isothermal at its saturation temperature. The vapor in the film between droplet and plate is assumed to be super heated to a temperature half away

The theoretical analysis does not require any experimental data (except physical properties) for the prediction of droplet evaporation time; however, it involves complicated iterative computations before it converges to the correct values for droplet evaporation 
time. For engineering calculation it is desirable to obtain a relatively simple equation which would imply the correct functional dependence upon variables and allow a prediction for droplet evaporation time without recourse to a computer. In order to obtain such an empirical correlation of the experimental data, a functional equation between the dependent variables and the independent variables must be obtained.

From the theoretical development, it is found that heat is transferred from the plate to the droplet by conduction and radiation, neither one of which may be neglected in general. The evaporation rate per unit area for a spherical droplet is on the order of $\rho_{\mathrm{L}} \mathrm{r}_{\mathrm{o}} / \tau$ and this quantity is equal to the sum of the heat transferred by conduction and radiation divided by $\lambda$ '. Functional arguments are developed in detail in (Gottfried et al., 1966), but the resulting equation is:

$$
\frac{\rho_{\mathrm{L}} \mathrm{r}_{\mathrm{o}}}{\tau}=\mathrm{C}_{1}\left[\frac{\mathrm{k} \Delta \operatorname{Tr}_{\mathrm{o}} \mathrm{g} \rho_{\mathrm{V}}\left(\rho_{\mathrm{L}}-\rho_{\mathrm{V}}\right)}{\mu \lambda^{\prime}}\right]^{\frac{1}{2}}+\mathrm{C}_{2}\left[\frac{\sigma \varepsilon_{\mathrm{P}}\left(\mathrm{T}_{\mathrm{P}}^{4}-\mathrm{T}_{\mathrm{S}}^{4}\right)}{\lambda^{\prime}}\right]
$$

Where:

$\rho_{\mathrm{L}}=$ Density of saturated liquid $\left(\mathrm{g} \mathrm{cm}^{-3}\right)$

$\rho_{\mathrm{V}}=$ Density of vapor $\left(\mathrm{g} \mathrm{cm}^{-3}\right)$

$\mathrm{T}_{\mathrm{P}}=$ Plate temperature $(\mathrm{K})$

$\mathrm{T}_{\mathrm{S}}=$ Saturation temperature of liquid droplet $(\mathrm{K})$

$\mu=$ Viscosity of vapor at $T_{V}\left(\mathrm{~g} \mathrm{~cm}^{-1} \mathrm{sec}\right)$

$\mathrm{T}_{\mathrm{V}}=$ Mean vapor temperature beneath $\operatorname{droplet}\left({ }^{\circ} \mathrm{C}\right)$

$\mathrm{r}_{\circ}=$ Initial radius of droplet $(\mathrm{cm})$

$\tau=$ Total droplet evaporation time (sec)

$\lambda^{\prime}=\lambda+(\Delta \mathrm{T} / 2) \mathrm{C}_{\mathrm{p}}\left(\mathrm{cal} \mathrm{g}^{-1}\right)$

$\lambda=$ Heat of vaporization of saturated liquid $\left(\mathrm{cal} \mathrm{g}^{-1}\right)$

$\sigma=$ Stefan-Boltzman constant, $\mathrm{C}_{1}$ and $\mathrm{C}_{2}$ are constants to be evaluated from the experimental data

The first and second part of the above equation represents the conduction and radiation heat transfer respectively.

Thirty three data points representing the full range of experimental condition were selected and used to estimate $\mathrm{C}_{1}$ and $\mathrm{C}_{2}$ by least squares fitting.

The resulting correlation for four liquids on aluminum is:

$$
\begin{aligned}
\frac{\rho_{\mathrm{L}} \mathrm{r}_{\mathrm{o}}}{\tau}= & 0.0026\left[\frac{\mathrm{k} \Delta \operatorname{Tr}_{\mathrm{o}} \mathrm{g} \rho_{\mathrm{V}}\left(\rho_{\mathrm{L}}-\rho_{\mathrm{V}}\right)}{\mu \lambda^{\prime}}\right]^{\frac{1}{2}} \\
& +23.9\left[\frac{\sigma \varepsilon_{\mathrm{P}}\left(\mathrm{T}_{\mathrm{P}}^{4}-\mathrm{T}_{\mathrm{S}}^{4}\right)}{\lambda^{\prime}}\right]
\end{aligned}
$$
is:

The resulting correlation for four liquids on brass

$$
\begin{aligned}
\frac{\rho_{\mathrm{L}} \mathrm{r}_{\mathrm{o}}}{\tau}= & 0.0002\left[\frac{\mathrm{k} \Delta \operatorname{Tr}_{\mathrm{o}} \mathrm{g} \rho_{\mathrm{V}}\left(\rho_{\mathrm{L}}-\rho_{\mathrm{V}}\right)}{\mu \lambda^{\prime}}\right]^{\frac{1}{2}} \\
& +41.8\left[\frac{\sigma \varepsilon_{\mathrm{P}}\left(\mathrm{T}_{\mathrm{P}}^{4}-\mathrm{T}_{\mathrm{S}}^{4}\right)}{\lambda^{\prime}}\right]
\end{aligned}
$$

The above equation shows that the Leidenfrost time is dominated by radiation heat transfer. In addition radiation heat transfer is more dominating at higher temperatures. The value of the coefficient $C_{1}$ for both the Eq. 2 and 3 is very small. It indicates that the first group of the Eq. 1 is not so dominating for the correlation. In future study the correlating group will be re evaluated. The estimated Leidenfrost time from Eq. 2 and 3 are shown in Fig. 10 and 11 for Aluminum and Brass respectively. If the values of the time for each corresponding liquids are compared between Fig. 2 and 10 for Aluminum, a fair agreement is obtained for the experimental values Fig. 2 and predicted values from the correlation Fig. 10. Again, if the values of the time for each corresponding liquids are compared between Fig. 3 and 11 for Brass, a fair agreement is also obtained for the experimental values Fig. 3 and predicted values from the correlation Fig. 11.

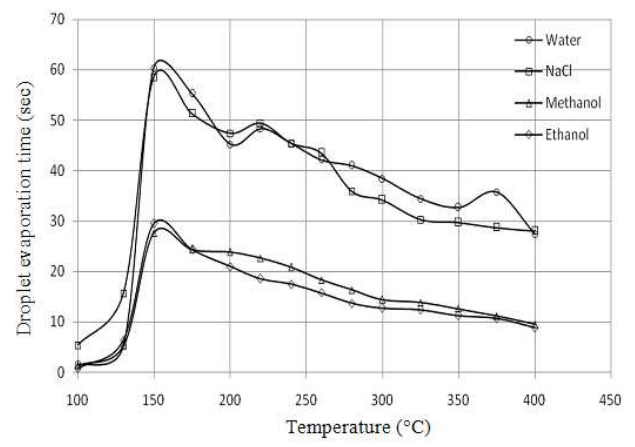

Fig. 10: Comparison of droplet evaporation time from Eq. 2 of water, $\mathrm{NaCl}$ solution, methanol, ethanol on aluminum

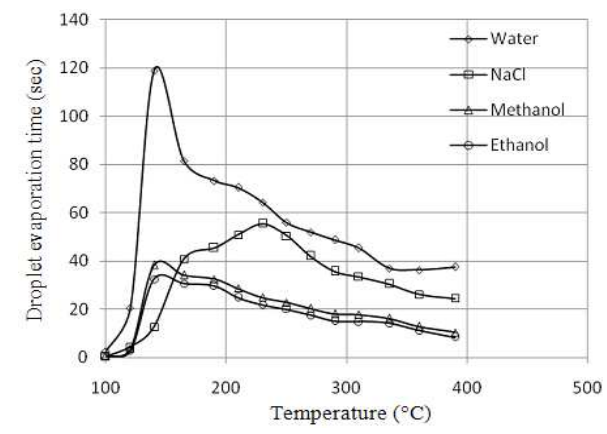

Fig. 11: Comparison of droplet evaporation time from Eq. 3 of water, $\mathrm{NaCl}$ solution, methanol, ethanol on brass 


\section{CONCLUSION}

Due to the involvement of many parameters, the Leidenfrost time and temperature become a complicated phenomenon. More investigation is indispensable to have a clear picture for this important feature of heat transfer. A mathematical model and well organized correlation have yet to be derived. The fundamental understandings at the moment are summarized as below:

- Sessile drop evaporation time is the maximum for water, then decreases gradually for Nacl solution, methanol and is the minimum for ethanol for a particular solid material

- On the other hand, the Leidenfrost time is the highest for copper and the lowest for mild steel for a specific liquid (there is some exception also)

- The radiative heat flux dominates the heat transfer process beyond the Leidenfrost temperature

- The experimental data for the evaporation time fairly agree with the proposed correlation within a certain range

\section{ACKNOWLEDGMENT}

The researcher acknowledges their gratefulness to mechanical engineering department, BUET for providing required facilities. The authors are also thankful to instructor, operators and Technicians of different shops and laboratories for their help during the fabrication of the experimental setup.

\section{REFERENCES}

Bernardin, J.D. and I. Mudawar, 1999. The Leidenfrost point-experimental study and assessment of existing models. J. Heat Transfer., 121: 894-903.

Gottfried, B.S., C.J. Lee and K.J. Bell, 1966. The Leidenfrost phenomenon: Film boiling of liquid droplets on a flat plate, Int. J. Heat Mass Transfer, 9: 1167-1187.

Elyssa, F.C. and W.Z. Black, 2004. Heat transfer and evaporation rates of small liquid droplets on heated horizontal surfaces. Int. J. Heat Mass Transfer, 47: 1187-1200.

Michiyoshi, I. and K. Makino, 1978. Heat transfer characteristics of evaporation of a liquid droplet on heated surfaces. Int. J. Heat Mass Transfer, 21: 605-613.

Nikolopoulos, N., A. Theodorakakos and G. Bergeles, 2007. A numerical investigation of the evaporation process of a liquid droplet impinging onto a hot substrate. Int. J. Heat Mass Transfer, 50: 303-319.

Heng, X. and Z. Zhou, 2007. A model for droplet evaporation near Leidenfrost point. Int. J. Heat Mass Transfer, 50: 5328-5333. 